\title{
A NECESSARY AND SUFFICIENT CONDITION THAT A FUNCTION ON THE MAXIMAL IDEAL SPACE OF A BANACH ALGEBRA BE A MULTIPLIER
}

\author{
JAMES A. WOOD
}

\begin{abstract}
ABSTRaCr. Consider a regular commutative, semisimple Banach algebra with a bounded approximate identity whose Gelfand transforms have compact support. A necessary and sufficient condition is given for a complex valued function defined on the maximal ideal space to determine a multiplier of the algebra. This theorem is similar to one proved by $F$. T. Birtel, but omits Birtel's assumption that the algebra be topologically embeddable in its second dual.
\end{abstract}

1. Introduction. Let $A$ be a regular commutative semisimple Banach algebra and denote by $\Delta(A)$ the maximal ideal space of $A$ endowed with the Gelfand topology. If $x \in A, \hat{x}$ will denote the Gelfand transform of $x$ and $\hat{A}$ the algebra of all transforms. We assume further that $A$ has a bounded approximate identity $\left\{e_{n}\right\}$ and that each $\hat{e}_{n}$ has compact support. By a multiplier of $A$ we mean a bounded linear operator $F: A \rightarrow A$ such that $F(x y)=x F(y)$ for all $x, y \in A$.

If $\hat{F}$ is a complex valued function on $\Delta(A)$, we denote by $A_{F}$ the set $\{x \mid \hat{F} \hat{x}=\hat{y}$ for some $y \in A\}$. The set $A_{F}$ is always nonempty since it contains at least $x=0$. Moreover, $A_{F}$ is a subspace of $A$, and we can define a linear function $F: A_{F} \rightarrow A$ by $F(x)=y$ if and only if $\hat{F} \hat{x}=\hat{y}$. If $A_{F}=A$, then an application of the closed graph theorem shows that $F$ is a bounded linear operator, and it is obvious that $F$ is a multiplier of $A$. Conversely, it is also well known that if $F$ is a multiplier of $A$, then there is a unique function $\hat{F}$ on $\Delta(A)$ such that $F(x)^{\wedge}=\hat{F} \hat{x}$ for all $x$. This correspondence is actually an isomorphism.

It is the purpose of this note to prove a necessary and sufficient condition that a function $\hat{F}$ on $\Delta(A)$ determine a multiplier $F$ of $A$. This theorem is related to a theorem proved by F. T. Birtel in 1962 in [2, p. 819]. The main difference between our result and Birtel's theorem is that we are able to replace Birtel's assumption that $A$ be topologically embeddable in $A^{\prime \prime}$ with a different assumption which does not involve $A^{\prime \prime}$. Here $A^{\prime \prime}=\left(A^{\prime}\right)^{*}$, where $A^{\prime}$ is the closed linear span of $\Delta(A)$ in $A^{*}$, the conjugate space of $A$.

In what follows we need to make use of the fact that $A^{* *}$ is a Banach

Received by the editors July 13, 1976 and, in revised form, December 27, 1976 and January 27, 1977.

AMS (MOS) subject classifications (1970). Primary 46J99. 
algebra under a multiplication introduced by R. Arens [1], or [2, p. 816]. For the sake of completeness, we sketch the definition of this multiplication. If $p \in A^{*}, x \in A$, define $p x(y)=p(x y)$. It is easy to check that $p x \in A^{*}$ and $\|p x\| \leqslant\|p\|\|x\|$. If $\Phi \in A^{* *}, p \in A^{*}$, define $\Phi p$ by $\Phi p(x)=\Phi(p x)$. Again it is easy to check that $\Phi p \in A^{*}$ and $\|\Phi p\| \leqslant\|\Phi\|\|p\|$. Therefore, if $\Phi, \Psi \in$ $A^{* *}$, define $\Phi \Psi(p)=\Phi(\Psi p), p \in A^{*}$. Finally, $\Phi \Psi$ is linear on $A^{*}$ and $\|\Phi \Psi\| \leqslant\|\Phi\|\|\Psi\|$, so $\Phi \Psi \in A^{* *}$.

2. Proof of the Main Theorem. Before proving the main theorem we first need a lemma.

LEMMA. Suppose $\hat{F}$ is a complex valued function defined on $\Delta(A)$ and that for some $x \in A, \hat{F} \hat{x} \in \hat{A}$. Let $y$ be that element in $A$ such that $\hat{y}=\hat{F} \hat{x}$ and write $y=F(x)$. Assume $|p(y)| /\|p x\|=|p(F(x))| /\|p x\| \leqslant M$ for all $p \in A^{*}$. Then there exists a $\Phi \in A^{* *}$ such that $y^{* *}=(F(x))^{* *}=\Phi x^{* *}$, where the product $\Phi x^{* *}$ denotes the Arens product in $A^{* *}$.

Proof. Let $x^{* *} A^{*}=\left\{x^{* *} p \mid p \in A^{*}\right\}$. It is routine to check that $x^{* *} p+$ $x^{* *} q=x^{* *}(p+q)$ and that $\alpha\left(x^{* *} p\right)=x^{* *} \alpha p$ so that $x^{* *} A^{*}$ is a subspace of $A^{*}$. We define $\Phi_{0}$ on $x^{* *} A^{*}$ by $\Phi_{0}\left(x^{* *} p\right)=p(F(x))$. Now

$$
\begin{aligned}
\Phi_{0}\left(x^{* *} p+x^{* *} q\right) & =\Phi_{0}\left(x^{* *}(p+q)\right)=(p+q)(F(x)) \\
& =p(F(x))+q(F(x))=\Phi_{0}\left(x^{* *} p\right)+\Phi_{0}\left(x^{* *} q\right) .
\end{aligned}
$$

Also

$$
\Phi_{0}\left(\alpha x^{* *} p\right)=\Phi_{0}\left(x^{* *} \alpha p\right)=\alpha p(F(x))=\alpha \Phi_{0}\left(x^{* *} p\right) .
$$

Thus $\Phi_{0}$ is linear on $x^{* *} A^{*}$. Moreover,

$$
\frac{\left|\Phi_{0}\left(x^{* *} p\right)\right|}{\left\|x^{* *} p\right\|}=\frac{|p(F(x))|}{\|p x\|} \leqslant M,
$$

so $\left\|\Phi_{0}\right\| \leqslant M$ and $\Phi_{0}$ is bounded. By the Hahn-Banach theorem $\Phi_{0}$ can be extended to a functional $\Phi$ on all of $A^{*}$ having the same norm as $\Phi_{0}$. Now

$$
(F(x))^{* *}(p)=p(F(x))=\Phi_{0}\left(x^{* *} p\right)=\Phi\left(x^{* *} p\right)=\Phi x^{* *}(p)
$$

for all $p \in A^{*}$, so that $(F(x))^{* *}=\Phi x^{* *}$.

We can now prove our main result.

THEOREM. Let $\hat{F}$ be a complex valued function on $\Delta(A)$. In order that $\hat{F}$ determine a multiplier of $A$, it is necessary and sufficient that $\hat{F}$ belongs locally to $\Delta(A)$ at each point of $\Delta(A)$ and that $|p(F(x))| /\|p x\| \leqslant M$, for all $p \in A^{*}$ and for all $x \in A_{F}$.

Proof. We show first that the condition is sufficient. In [2, p. 818], Birtel showed that if a function $f$ on $\Delta(A)$ belongs locally to $\hat{A}$ at each $p \in \Delta(A) \cup$ $\{\infty\}$, then $f \in \hat{A}$. From this result it follows that $\hat{F} \hat{x} \hat{e}_{n} \in \hat{A}$ for all $x \in A$ and all $n$, i.e. $x e_{n} \in A_{F}$. By the lemma, we know that $\left(F\left(x e_{n}\right)\right)^{* *}=\Phi_{n m}\left(x e_{n}\right)^{* *}$, where $\left\|\Phi_{n m}\right\| \leqslant M$ and $M$ is . independent of $x$ and $\Phi$ in general depends upon $F$ and $x e_{n}$. Thus 


$$
\begin{aligned}
\left\|F\left(x e_{n}\right)-F\left(x e_{m}\right)\right\| & =\left\|\left(F\left(x e_{n}\right)-F\left(x e_{m}\right)\right)^{* *}\right\| \\
& =\left\|\Phi_{n m}\left(x e_{n}-x e_{m}\right)^{* *}\right\| \\
& \leqslant\left\|\Phi_{n m}\right\|\left\|\left(x e_{n}-x e_{m}\right)^{* *}\right\| \\
& \leqslant M\left\|x e_{n}-x e_{m}\right\| .
\end{aligned}
$$

Therefore, $\left\{F\left(x e_{n}\right)\right\}$ is a Cauchy net in $A$. Let $y=\lim F\left(x e_{n}\right)$ and define $F(x)=y$. Clearly, $\hat{F} \hat{x}=\hat{y}$ so that $\hat{F}$ determines the multiplier $F$.

To prove the necessity suppose $\hat{F}$ determines the multiplier $F$. It is an easy consequence of the regularity of $A$ that $\hat{F}$ belongs locally to $\hat{A}$ at points of $\Delta(A)$. To obtain the desired estimate we observe first that

$$
\begin{aligned}
\left|p\left(F\left(x e_{n}\right)\right)\right| & =\left|p\left(x F\left(e_{n}\right)\right)\right|=\left|p x\left(F\left(e_{n}\right)\right)\right| \\
& \leqslant\|p x\|\|F\|\left\|e_{n}\right\| \leqslant\|p x\| M .
\end{aligned}
$$

Therefore, $\left|p\left(F\left(x e_{n}\right)\right)\right| /\|p x\| \leqslant M$. But

$$
p\left(F\left(x e_{n}\right)\right)=p\left(e_{n} F(x)\right) \rightarrow p(F(x))
$$

so that $|p(F(x))| /\|p x\| \leqslant M$ for all $x \in A$ and all $p \in A^{*}$.

\section{REFERENCES}

1. R. Arens, Operations induced on function class, Monatsh. Math. 55 (1951), 1-19.

2. F. T. Birtel, On a commutative extension of a Banach algebra, Proc. Amer. Math. Soc. 13 (1962), 815-822.

Department of Mathematics, Virginia Commonwealth University, Richmond, Virginia 